\title{
Energy loss researches of diamond microgrinded laser mirrors by Fourier optics methods
}

\author{
V.S. Staschuk, G.L. Kononchuk, L.V. Poperenko, V.V. Stukalenko, Y.V. Filipov \\ Taras Shevchenko Kyiv National University, Department of Physics, \\ 6, prospect Glushkova, 03127 Kyiv, Ukraine; \\ E-mail:stu@univ.kiev.ua; filipov@univ.kiev.ua
}

\begin{abstract}
The diffraction losses of laser mirrors were determined by using specially designed spectrometer of spatial frequencies. Reflection surface (70 mm diameter) was made by diamond microgrinding, but instead of an ideal plane, the circular phase grating was formed. The Fourier-optics was applied to measure a real shape of flat laser metal mirrors. We analysed the diffraction pattern of light reflected from metal mirror in the Fraunhofer zone and determined periodic deviation of a surface from an ideal plane and its parameter - spatial frequency.
\end{abstract}

Keywords: metal mirror for laser, energy losses, Fourier-optics method, circular phase grating, spatial frequency.

Manuscript received 11.11.08; revised manuscript received: 28.04.09; accepted for publication 14.05.09; published online 29.05.09.

Development of a new technology to produce high quality metal mirrors with the reflection coefficient value higher than $R \approx 0.99$ is a serious problem in a powerful laser design [1,2]. Even small diffraction losses have the same negative influence as transmission losses for a laser resonator with these mirrors. The energy losses can be caused by deformation of the reflection surface structure during metal shaping. That deformation influences both on power and spatial characteristics of radiation $[3,4]$. The most important characteristic of high quality laser mirrors is the minimum of energy losses in reflection, including the diffraction ones, too.

For diagnostics of a metal mirror surfaces, a few optical methods can be used. One of them is the shadow method. It can be specified by simplicity of acquired visual data about mirror surface. But quantitative data about surface shape cannot be acquired from shadow method results. Another one is the Hartmann method that after appropriate handling of initial results allows us to acquire visionary as well as quantitative data. Unfortunately, one of the main disadvantages is a low dimensional resolution capability for both of these methods. Therefore, for detection and describing a small-scale deformation of a laser mirrors surface, the interferometric method was chosen. Fourier-optics methods [5-7] find a wide application as a solution method for different scientific and applied tasks. The principle of this method is to get some certain function spectrum and then to reproduce that unknown function by spectrum handling. Fourier-optics methods are very sensible to the surface shape. Thus, they can be used for research of surface quality, in particular case, for surfaces of laser metal mirrors.

There a few main methods to make a reflection surface - polishing, grinding, turning, etc. Better purity of surface is obtained by the diamond microgrinding of a metal specimen. But a grinding process modifies shape from an ideal plane by cutter track. Moreover, surface of a mirror retains phase modulation even after deposition of a coating with reflection up to $100 \%$. Hence, almost always there are diffraction losses in light reflected from laser metal mirrors made by method of a diamond microgrinding. The light diffraction pattern in the Fraunhofer area explicitly depends on spatial parameters of the grating on which light diffracts. Thus, control and analysis of a diffraction influence on reflection allow making a proposal for further decreasing the small-scale deformations of surface made by diamond microgrinding.

The objects of research are flat round copper and aluminium mirrors with $70 \mathrm{~mm}$ diameter. Reflection surfaces are made by a diamond microgrinding of a metal specimen. Metal surface of investigated specimens are made by galvanic or vacuum deposition of a metal layer with $250-300 \mu \mathrm{m}$ thickness on a ceramic. Vacuum deposition was carried out with controlled variation of a high-energy electron beam at the surface of evaporated metal. Galvanic deposition was carried out in special tank with a sulphuric electrolyte that contained salt of 
required metal. Several modes for deposition were used - variation of the current intensity, mixing of electrolyte in the ultrasonic tank and others. Selected specimens were annealed at high temperature after galvanic deposition. Finally, the reflecting surface was shaped by diamond microgrinding with fixed cutting depth of $5 \mu \mathrm{m}$ and speeds of cutter serve of 5 and $10 \mu \mathrm{m}$. Diamond microgrinding is the method to produce high quality metal mirror surfaces, it allows on-line handling of required geometry of metal mirror surface. Moreover, the surfaces with the parameters of roughness $R_{\max } \leq 0.01 \mu \mathrm{m}$ and deviation from predefined form less than $0.1 \mu \mathrm{m}$ can be produced while increasing the productivity by $10-50$ times [8].

In theory, such surface would be absolutely flat because of orthogonality of rotation axis and motion direction of cutter. However, the mirror surface is not absolutely flat as a result of mutual vibrations between instrument and specimen during the grinding process. The surface has defects: concentric rings, cavities and humps. In our particular case, this circular structure has regular character (mutual vibrations of detail and instrument of grinding machine in the automatic mode are stable during processing), and a circular phase diffraction grating appears on surface. The period of circular structure gives information about the frequency and source of mutual vibrations. The amplitude of circular structure (deviation from an ideal plane) is proportional to the amplitude of mutual vibrations of specimen and cutter during a grinding. This value contains information about quality of the technological process.

Thus, a flat laser metal mirror with a circular structure can be considered as a planar reflective phase grating with concentric grooves. Hence, it is possible to find relations between diffraction patterns of light in a far area and the spatial characteristic of this grating. Let's consider a phase modulation of a plane wave reflected from a mirror:

$$
E=E_{0} e^{i \varphi_{0} \sin p r},
$$

where $E_{0}$ is the value of electric field of initial wave, $\varphi_{0}=2 \pi \frac{2 \Delta x_{0}}{\lambda}$ is the amplitude of wave phase modulation, $\Delta x_{0}$ is the maximal deviation of mirror surface from an average plane position, $\lambda$ is the wavelength of light, $p=\frac{2 \pi}{T}$ is the spatial frequency, and $T$ is the modulation period along the radius of mirror.

Fourier transformation of the function (1) with assumption of system axial symmetry:

$G(\Omega)=\frac{e^{i \frac{2 \pi}{\lambda} F}}{i \lambda F} \int_{0}^{n_{0}} E_{0} e^{i \varphi_{0} \sin p r} J_{0}(r \Omega) r d r$.

Here, $F$ is the focal distance of a lens, $2 r_{0}$ is the beam (or mirror) diameter, $\Omega=2 \pi \frac{\rho}{F}$ is the spatial frequency, $\rho$ is a radial coordinate in the screen plane, $J_{0}(r \Omega)$ is the zero order Bessel function, and $G(\Omega)$ is a distribution of explored field density in the lens focal plane. This equation (2) determines a spectrum position in the Fraunhofer range in the case of symmetric object. The formula consists of two parts: first (before the integral sign) is the ordinary term describing the wave amplitude and phase in the lens focal plane, second term (integral) - describing a field distribution in a lens focal plane as dependent on the object character - distribution of the intensity along the radius. The spectrum of our object can be found applying the Fourier-Bessel transformation, because our objects have axial symmetry [9].

However, in our case of weak modulation $\left(\varphi_{0}<<1\right)$ an influence of phase modulation is similar to that of amplitude modulation [10]. It means that the energy of an incident wave forms three characteristic beams after diffraction on the grating. The first beam propagates without a change of initial direction and has the energy

$W_{0}\left(1-\frac{\varphi_{0}^{2}}{2}\right)$.

Two other beams propagate as diffraction peaks with a vertex of cone $\pm 2 \arcsin \left(2 \pi \frac{\lambda}{T}\right)$, and each of them has the energy $W_{0} \varphi_{0}^{2} / 4$. However, these beams absolutely coincide in space because of symmetry of the system.

The principal scheme of the experimental device is shown in Fig. 1. As a light source, we used $1 \mathrm{~mW}$ helium-neon laser LGN-208 with $632.8 \mathrm{~nm}$ wavelength; the light detector is a photomultiplier FEU-68; beam splitter is a plane-parallel glass plate with multilayer coating; beam shaping system is a telescopic system based on objective OF-41 with a beam diameter $85 \mathrm{~mm}$ and a focal length $400 \mathrm{~mm}$. This telescopic system,

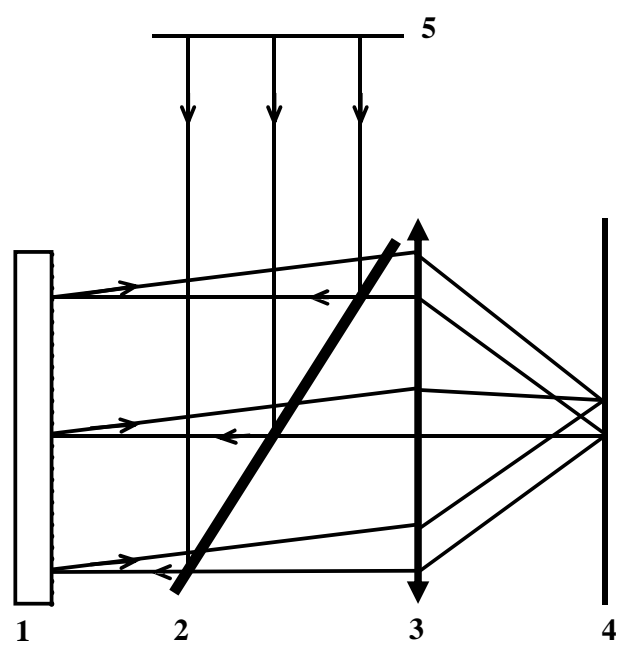

Fig. 1. The principal scheme of the experimental device: 1 - laser mirror; 2 - beam splitter; 3 - lens; 4 - detector; 5 - light source. 


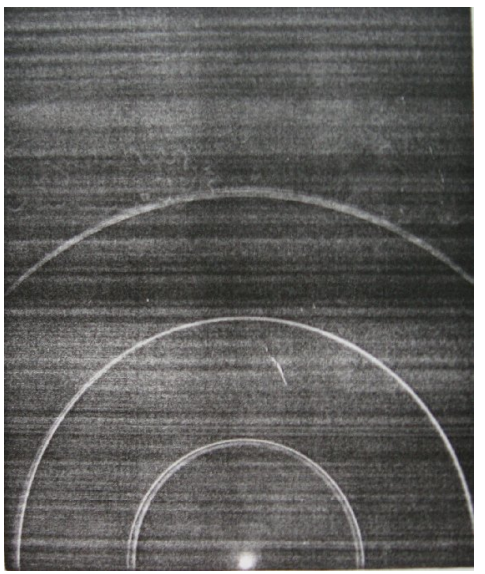

a

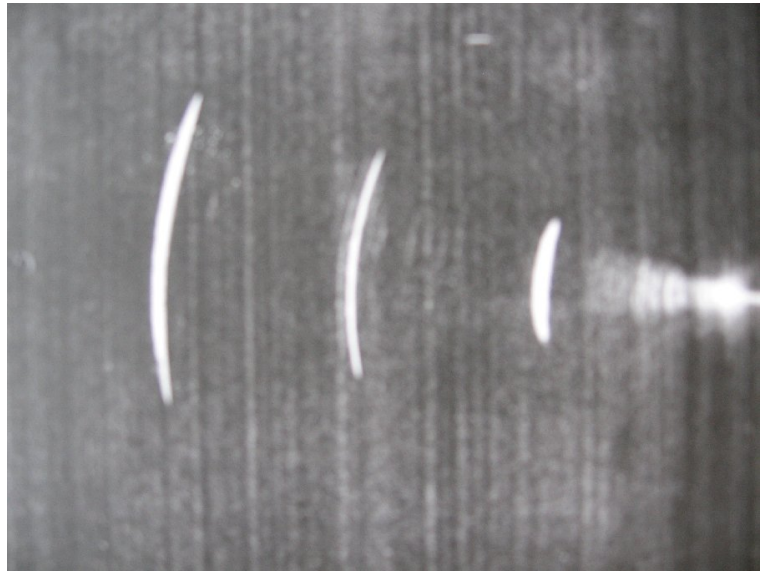

b

Fig. 2. Photos of examples of the diffraction patterns for the laser metal mirror. Axes of symmetry of the mirror and lens (see Fig. 1) coincide (a) or are slightly shifted (b).

Table

\begin{tabular}{|c|c|c|c|c|c|c|c|c|c|}
\hline $\begin{array}{l}\text { The number } \\
\text { of a mirror }\end{array}$ & \multicolumn{8}{|c|}{ Spatial frequency, $\mathrm{cm}^{-1}$} & \multirow{2}{*}{$\begin{array}{l}\text { Diffraction losses, } \\
\beta \times 10^{2} \\
1.43\end{array}$} \\
\hline 1 & 50 & 150 & 200 & 300 & - & - & - & - & \\
\hline 2 & 50 & 200 & 300 & 400 & 600 & - & - & - & 1.14 \\
\hline 3 & 50 & 150 & 280 & 310 & - & - & - & - & 0.62 \\
\hline 4 & 50 & 120 & 330 & 370 & - & - & - & - & 0.29 \\
\hline 5 & 50 & 100 & 320 & 380 & 650 & 700 & 880 & 900 & 0.62 \\
\hline 6 & 80 & 200 & 280 & 300 & 450 & 550 & 600 & - & 0.83 \\
\hline 7 & 80 & 150 & 300 & 380 & 1005 & 2150 & - & - & 0.64 \\
\hline 8 & 100 & 150 & 300 & 450 & 780 & - & - & - & 0.43 \\
\hline 9 & 80 & 140 & 300 & 400 & 650 & 730 & 830 & 1002 & 0.40 \\
\hline 10 & 60 & 140 & 250 & 300 & 730 & 900 & 1002 & 1100 & 0.37 \\
\hline
\end{tabular}

which increases beam size to $40 \mathrm{~mm}$, forms the plane wave front 5 . There is an optical detector FEU-68 with a small aperture in the screen plane. Shown in Fig. 2 are photos of examples of the diffraction pattern on specified metal mirrors, and in Fig. 3 there is an example of the photo-electric registration. To register the highfrequency components of a spatial frequency spectrum for the beam reflected from the mirror, an axis misalignment is required. To be exact, beams don't match in lens 3 after reflection from the mirror 1 within this coaxial scheme. Therefore, the lens 3 should be shifted from the axis of the mirror 1 , and symmetry of the picture in the focal plane disappears.

As can be seen from the figures, in light reflected from a mirror one can observe a central diffraction maximum and a few other (lateral) peaks from diffracted waves. In our approximation $\left(\varphi_{0}<<1\right)$, there is only one first order maximum. Therefore, we can suppose that these few lateral maxima are caused by a few sinusoidal diffraction gratings with different spatial periods $T$.

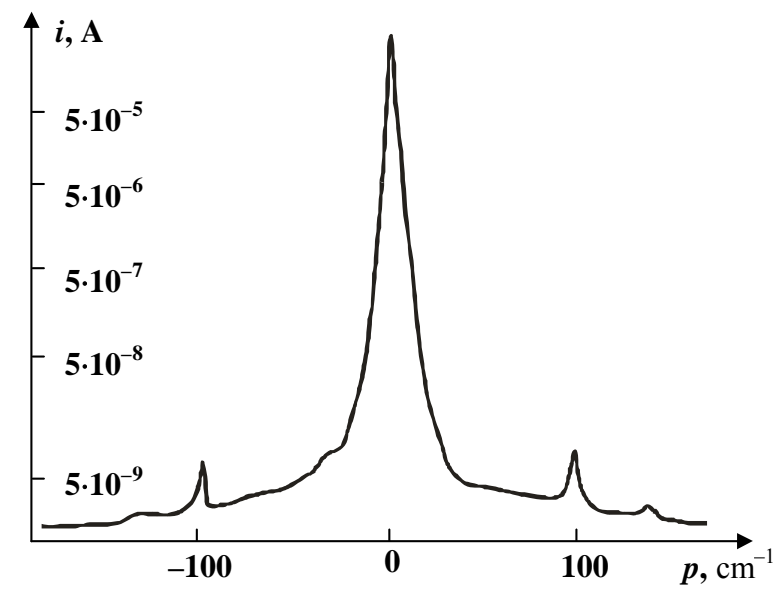

Fig. 3. The diffraction spectrum registered by PMU. X-axis is the spatial frequencies, Y-axis is an output signal of detector. 
The total dispersion energy in $i$-ring is obtained by integrating:

$$
W_{i}=2 \pi \int_{0}^{r_{0}} \rho G^{2}\left(2 \pi \frac{\rho_{i}}{F}\right) d \rho,
$$

where $i$ means the number of a diffraction $\operatorname{ring}(i=$ $1,2,3 \ldots)$, and $G^{2}\left(2 \pi \frac{\rho}{F}\right)$ is the radial distribution of the light intensity in a ring. That distribution can be acquired from an experimental data. The coefficient of diffraction losses is obtained in the following way:

$\beta=\sum_{i=1} W_{i} / \sum_{i=0} W_{i}$,

where $W_{i}$ is the light intensity in the $i$-th order of diffraction.

The diffraction losses of light reflected from mirrors with phase modulation of surface and the spatial frequency values of the laser metal mirrors made by diamond microgrinding are given in Table. The proposed method of interferometric analysis allows to find mechanical vibration frequencies and quantitatively describe defects of mirror surface. After appropriate handling the obtained data, it is possible to define and eliminate sources of vibrations.

As a result, application of the Fourier transformation to the diffraction pattern from metal mirrors allowed:

a) to determine the deviation level of a mirror surface from an ideal plane and parameters of periodic components - amplitude and spatial frequencies;

b) to experimentally determine the diffraction losses of laser metal mirrors by the specially designed spectrometer of spatial frequencies.

The results of such researches have an applied importance, as they make a basis for numerous applications of lasers in science and industry.

\section{References}

1. V.P. Koronkevich and G.A. Lenkova, Features of the interference field in a diffraction interferometer for surface testing // Optika i spektroskopiya 99(1), p. 168-175 (2005) (in Russian).

2. D.L. Carrol, L.H. Sentman, Maximizing output power of a low-gain laser system // Appl. Opt. 32(21), p. 3930-3941 (1993).

3. Y.A. Anan'ev, Optical Resonators and Laser Beams. Mir Publ., Moscow, 1990 (in Russian).

4. Emil Wolf, Introduction to the Theory of Coherence and Polarization of Light. University Press, Cambridge, 2007.

5. A.M. Lyalikov, Determination of a surface macrorelief with the double-exposure fringe projection method // Optika i spektroskopiya 96(3), p. 523-527 (2004) (in Russian).

6. Joseph W. Goodman, Introduction to Fourier Optics. Mir Publ., Moscow, 1970 (in Russian).

7. G.N. Vishnyakov, G.G. Levin, K.E. Loshchilov, K.A. Sukhorukov, Fourier synthesis of a threedimensional surface by the method of multiangle projection of fringes // Optika $i$ spektroskopiya 99(4), p. 654-658 (2005) (in Russian).

8. L.V. Poperenko, V.S. Staschuk et al., Optical investigation surface of bulk copper mirrors // Visnyk Kyivs 'kogo Natsional'nogo Universytetu im. T.G. Shevchenko 7, p. 54-56 (2005) (in Ukrainian).

9. A. Papulis, Theory of Systems and Conversion in Optics. Mir Publ., Moscow, 1971 (in Russian).

10. S.I. Baskakov, Radio Circuits and Signals. Vysshaya Shkola, Moscow, 1983 (in Russian). 